\title{
A Grand Challenge-based Framework for Contextual Learning in Engineer- ing
}

\section{Dr. Lisa G. Huettel, Duke University}

Dr. Lisa G. Huettel is an associate professor of the practice in the Department of Electrical and Computer Engineering at Duke University where she also serves as associate chair and director of Undergraduate Studies for the department. She received a B.S. in Engineering Science from Harvard University and earned her M.S. and Ph.D. in Electrical Engineering from Duke University. Her research interests are focused on engineering education, curriculum and laboratory development, and applications of statistical signal processing.

\section{Dr. Michael R. Gustafson II, Duke University \\ Dr. Joseph C. Nadeau, Duke University \\ David Schaad, Duke University}

\begin{abstract}
Dr. David Schaad has over seventeen years of design and engineering experience as a consulting engineer working for various firms including: Parsons Engineering Science, Appian Consulting Engineers and Marshall Miller and Associates. As part of his experience, Dr. Schaad has: designed waste water treatment systems to address industrial and domestic waste streams; developed designs of storm water control structures and strategies to address water quality and quantity; designed fluid transport systems to replace water supplies impacted by anthropogenic sources; designed fuel transport and delivery systems; developed designs for commercial and residential development; prepared land use plans; developed designs to protect against potential flood hazards; designed and developed plans and specifications for fluid handling systems, waste mitigation alternatives and remedial actions for RCRA and CERCLA sites including active industrial facilities and inactive disposal sites (including NPL sites); conducted feasibility studies by evaluating and analyzing the economic and engineering considerations of multiple design alternatives; obtained extensive experience with innovative remedial techniques (including groundwater extraction and treatment, air sparging, soil vapor extraction, and bioventing). Current research focuses on sustainable engineering, community development, water and wastewater treatment design, stormwater retention/detention and treatment design, urban hydrology, constructed wetland and stream restoration design, ecological stabilization, sustainable engineering in land development, water resources, water and wastewater treatment. He is also the faculty advisor for Duke Engineers for International Development and the Duke Chapter of Engineers Without Borders and has led DukeEngage experiences every year since the inception of the program. He has facilitated and/or led trips to Indonesia, Uganda, Kenya, Honduras, El Salvador, Bolivia, and Peru. Representative projects he has worked on include: building a 4800sf Infant and Maternal Health Clinic, constructing a 100ft long vehicular bridge over a seasonally flooded river, and installing a $3 \mathrm{~km}$ long waterline. He was an inaugural member of the Faculty Leadership Council (FLC) of EWB and is a registered professional engineer in 21 states.
\end{abstract}

\section{Michael M Barger \\ Dr. Lisa Linnenbrink-Garcia, Duke University}

Dr. Lisa Linnenbrink-Garcia is an associate professor of Developmental Psychology and Education at Duke University. She received her Ph.D. in Education and Psychology from the University of MichiganAnn Arbor. Her research (1) identifies educational contexts that enhance motivation and subsequent engagement and learning and (2) examines the mechanisms through which motivation influences academic engagement and achievement. Dr. Linnenbrink-Garcia conducts this research in school-age samples (upper elementary through college), with a specific focus on students' learning and motivation in STEM fields. 


\title{
A Grand Challenge-based Framework for Contextual Learning in Engineering
}

\begin{abstract}
A key finding within the current engineering education literature is that exposure to real-world applications - especially when presented in an active, experiential learning environment increases both student interest and pedagogical effectiveness. This idea of contextual learning, learning designed so that students can carry out activities and solve problems in ways that reflect the real-world nature of such tasks, is based on cognitive learning theory and current research in cognitive psychology and neuroscience. Such research suggests that initial learning, transfer of that learning to other contexts, and retention of learned material is facilitated when concepts are presented in a familiar or relatable context. That is, students are motivated when learning has meaning.

The National Academy of Engineering recently outlined fourteen Grand Challenges for Engineering (e.g., "Reverse Engineering the Brain”) that collectively constitute some of the largest and most pressing real-world issues facing engineering research and practice. We have developed an instructional framework for engineering education that utilizes these Grand Challenges as the context for teaching a wide range of concepts. The framework comprises six stages, each building upon its predecessor. Students first progress from thinking about the grand, overarching theme from a multitude of perspectives to focusing on a discipline-specific view of the challenge (Stages 1-3). Then, they concentrate on learning the specific course content and applying what they have learned to a specific, real-world problem inspired by the overarching Grand Challenge (Stages 4-5). Finally, they analyze what they have learned from the hands-on activities and reflect back on how this can inform their understanding of, and solutions to, the Grand Challenge (Stage 6).

This paper begins with a description of the framework including its foundation in contextual learning theory and the motivation for using the Grand Challenges. Subsequently, the implementation of the framework in two engineering courses is described. Details of the learning modules and activities corresponding to the six stages of the framework are presented for each course. Similarities and differences in implementation are highlighted, illustrating how a common framework can be applied to seemingly very different courses. Finally, the use of the framework is evaluated in terms of its impact on student motivation and learning using psychologically rigorous measures.
\end{abstract}




\section{Introduction}

The National Academy of Engineering (NAE) has identified a set of fourteen Grand Challenges (GC) for current engineering research and practice. These include such diverse topics as reverseengineering the brain, providing access to clean water, and enhancing virtual reality. ${ }^{1}$ In its report, "Educating the Engineer of 2020," the NAE contends that solving problems, such as those posed in the Grand Challenges, will require more than just providing students with technical training. An engineering education must, it is argued, produce graduates who combine technical excellence with a multitude of other skills including communication, teaming, ethical reasoning, and contextual analysis. ${ }^{2}$ Yet, without exposure to real-world applications in the context of a technical education, students may neither develop these important skills nor gain sufficient motivation to pursue careers in engineering.

There are many successful examples of ways in which real-world problem solving has been integrated into engineering curricula: service learning (e.g., the EPICS program ${ }^{3}$ ), industrysponsored capstone design experiences, and cooperative learning internships. One common feature of these types of experiences is that they are often superimposed on top of a more traditional curriculum whose courses focus on fundamental engineering concepts. In ideal pedagogical practice, theory and application are tightly integrated so that students learn the fundamentals in the context of real-world problem solving throughout their entire academic experience. Such is the goal of techniques such as problem-based learning. However, identifying and integrating appropriate real-world problems into traditional courses, while still achieving very specific technical learning objectives, can be very daunting to many instructors. In this paper, we describe a pedagogical framework that facilitates the incorporation of key problems facing society, such as those described by the National Academy of Engineering's Grand Challenges for Engineering, into engineering curricula.

The multiple-stage framework is based on contextual learning theory, which espouses methods that link technical content to an applied context. Its stages progressively guide students from consideration of the overarching societal and technical context of a problem, through the discipline-focused exploration of a related application, and ultimately back to the broad problem context to analyze both the contributions and limitations of an engineering approach. We describe how this framework was incorporated into two courses in the Fall 2012 semester: 1) an introductory course on computational methods taken by all first-year engineering students, and 2) an upper-level Electrical and Computer Engineering elective in signal processing.

\section{Framework Description}

Meaningfully integrating real-world problems into a curriculum requires matching each problem (i.e., its statement and context) to specific topics within specific courses. Traditionally, instructors use relative narrow, discipline-specific problems. For example, in a digital signal processing course, students might design filters that manipulate audio signals; e.g., to remove distorting noise from a recording or to identify the frequency of notes emitted by plucked guitar strings. The Engineering Grand Challenge-based framework (which we will refer to as the EGC framework), is based on the use of broad, multi-disciplinary problems drawn from the NAE Grand Challenges for Engineering. Using these broad problems carries many advantages, from 
increased real-world relevance to broader contextual applications. However, it also introduces challenges: How can instructors distill a meaningful hands-on project from such a broad, overarching problem statement? How can students extract key concepts from each problem that have broader applicability to course material, specifically, and engineering, more generally? Incorporating diverse, interdisciplinary material may be daunting to many instructors. Thus, a primary goal of this project is to create a general framework for incorporating complex, crossdisciplinary problems, such as those described by the Grand Challenges, into individual, discipline-specific courses.

The instructional framework comprises six stages, each building upon its predecessor. Students first progress from thinking about the grand, overarching theme from a multitude of perspectives to focusing on a discipline-specific view of the challenge (Stages 1-3). Then, they concentrate on learning the specific course content and applying what they have learned to a specific, real-world problem inspired by the overarching Grand Challenge (Stages 4-5). Finally, they analyze what they have learned from the hands-on activities and reflect back on how this can inform their understanding of, and solutions to, the Grand Challenge (Stage 6).

The objectives and general implementation for each stage are described below. In the subsequent section, specific examples are provided in order to illustrate how this framework has been incorporated into two courses.

\section{Stage 1: Multi-Disciplinary Overview}

The students experience a multi-disciplinary overview of the specific Grand Challenge theme that (1) provides the overall context for later discipline-specific activities, (2) presents the Challenge from multiple perspectives within Engineering (e.g., societal context, policy, technical issues), and (3) gives examples of current efforts addressing the Challenge. The overview can take any of a number of forms: a live or recorded panel discussion that includes technical and non-technical experts (e.g., faculty researchers, policy makers), reading assignments that describe political or technical steps toward meeting the Challenge, and/or in-class discussions about ethical, practical, and social aspects of the Challenge. We emphasize that the manner in which this overview is presented can differ across institutions and courses, and based on the specific instructor's goals. In all cases, however, the broad, cross-disciplinary nature of the theme and its societal context should be emphasized.

\section{Stage 2: Definition/Problem Restatement}

Following the introduction of the theme, students reflect on what they have learned and then define the Challenge in their own words. This process engages the students as participants in the problem-solving process; they must progress from the relatively passive experience of Stage 1 to an active consideration of the Challenge (i.e., what makes it a difficult problem, what society and technical constraints exist?). This stage is critical for linking the overarching theme of the Challenge to the discipline-specific projects they will complete thereafter (Stage 5: Application). As this activity is one of reflection, drawing upon students' own personal/individual perspective, possible implementations may include writing a reflection paper that defines the key elements of the Challenge. Moreover, we note that this Stage provides a baseline for characterizing students' initial understanding of the Challenge to which their later understanding can be compared, for purposes of assessment. 


\section{Stage 3: Relation of the Grand Challenge to Engineering}

At this point, students have some understanding of both the technical challenges and the societal importance of the selected theme. Stage 3 begins the process of drilling down toward a discipline-specific problem that is related to the overarching challenge. Now, students will consider the question: Why is this an engineering Challenge? To achieve this goal, students need to be able to identify characteristics that define engineering problem solving generally (e.g., designing with constraints, optimization), analyze the specific Challenge from an engineering perspective, and form a link between the general and specific.

\section{Stage 4: Content, Tools, and Techniques}

Next, students learn course-specific content related to the discussion from the previous stage. This material can be taught using any pedagogical approach (e.g., lectures, in-class activities, computer simulations, discussion). It may be necessary to modify the course syllabus and/or method of presentation to ensure coherence between the course material and the theme. However, it is expected that these modifications, at least initially, could be minor. Although the methods used in this stage are similar to those typically used to incorporate real-world problems into a course, it is critical that they be well-integrated with the Grand Challenge theme.

\section{Stage 5: Application of Course Content to the Challenge}

Next, students apply the course-specific content, tools, and techniques to a real-world problem related to the Grand Challenge. Key elements of the application process include:

- Active, hands-on involvement of the student (e.g., through a laboratory or in-class exercise).

- Analysis of real-world data (e.g., climate data from an online database).

- Consideration of societal or technical constraints (e.g., cost-benefit tradeoffs).

If appropriate, local expertise and resources can be tapped to provide stimulating and relevant hands-on activities. These activities may include a visit to a research laboratory (e.g., a neuroscience laboratory), a local facility (e.g., the SmartHome), or a community resource (e.g., a local construction site). This approach facilitates links between the Challenge and the academic assignment, which is necessarily an abstraction of the real-world problem.

\section{Stage 6: Analysis and Reflection}

Finally, students return to a broad consideration of the Challenge itself. They reflect on how the specific application they explored in Stage 5 can inform solutions to the broader Challenge. Questions they might consider include: How does the analysis contribute to the solution of the Challenge? What are the limitations of the tools and techniques? What other factors and disciplines should be considered? They will also re-define the challenge (i.e., repeating Stage 2), so that the change in their understanding can be assessed.

\section{Framework Implementation}

The EGC framework was piloted in two courses in the Fall semester of 2012. These courses were at different levels (introductory required vs. advanced elective) and had very different 
content coverage. The following section describes how the framework was interpreted and implemented for each course.

\subsection{Computational Methods in Engineering}

Computational Methods in Engineering (EGR 103L) is required of all engineering students in their first semester. This course introduces students to computer methods and algorithms for the analysis and solution of engineering problems using numerical methods. For the Fall 2012 semester, the Grand Challenge theme Make Solar Energy Economical was incorporated into the course using the EGC framework. That theme was chosen in particular because of the rich tapestry of readily available data sets. Because EGR 103L is a course on computational methods, any theme which would provide data to be analyzed would work. In this particular case, however, the additional advantage of this theme being closely related to an institutional priority as well as the likelihood of some general understanding of solar energy made the theme more attractive than others. Specifically, the stages of the framework were implemented as follows:

\section{Stage 1 (Overview) and Stage 2 (Restatement)}

The students first considered the challenge of making solar energy economical in Homework $4{ }^{4}$ This assignment was introduced in a laboratory meeting during the $11^{\text {th }}$ week of the semester $(11 / 5 / 12)$ and due two weeks later. Stage 1 was accomplished through students' reading of the background material for the Grand Challenge as provided by the website for the National Academy of Engineering. One of the advantages to choosing the Grand Challenges for themes is, in fact, the presence of a ready-made multi-disciplinary overview that students can access on their own. One of the challenges for EGR 103L in using the framework is its size (over 350 students across three lecture sections with different instructors), so having this online resource was of tremendous benefit.

The students were then given the task of restating the problems involved with the challenge. Specifically, they had to summarize each of three different interviews about making solar energy economical; they had to find and discuss a reference that focused on a non-engineering aspect of the challenge; and they had to invent a set of hypothetical studies whose results would shed light on the grand challenge itself. The main purposes for this task were to make sure the student could communicate the interdisciplinary nature of the challenge and to make sure the student had some broader background for future analysis of specific components of the challenge.

\section{Stage 3 (Relation to Engineering) and Stage 4 (Content, Tools, and Techniques)}

Once the students turned in their papers, one laboratory session was set aside to discuss the relationship between the Grand Challenge and the course materials. Though this stage happened during the $14^{\text {th }}$ week of the semester $(11 / 26 / 12)$, earlier assignments had been altered or added specifically to motivate this discussion. Given that the Stage 5 application was to involve loading and manipulating data sets, as well as some economic analysis, an earlier assignment ${ }^{5}$ (Lab 4, problems 4.4.2 and 4.4.3) was changed in order to provide a foundation for the work required in the Stage 5 application. Through other labs, students had seen how to load and plot data, how to perform numerical integration, and how to find statistical measures of goodness of fit for a data set and a model. During the in-lab discussion in the $14^{\text {th }}$ week of the semester, students accessed the data sets for solar energy that come from a collection site in North Carolina and received 
instruction on how they would use the tools of EGR 103L to manipulate and analyze the data as well as present their results.

For Stage 4, the remainder of the time in lab was spent discussing the particular methods from class that would be used to work on the data and what kind of presentation would be required. Because EGR 103L is a general computational methods course, tying this assignment to a particular theme gave structure to the lab, but did not require reducing or eliminating the discussion of any other course material. In that way, a course such as EGR 103L is a perfect candidate for the EGC framework.

\section{Stage 5 (Application)}

This stage is captured in the students' work for Lab $10 .^{6}$ The students had to identify and download a data set that contained solar energy levels for four years. They then fit a sinusoidal model and analyzed the goodness of fit graphically and numerically (which tied in to several previous laboratory exercises: the graphical analysis of Lab 2, the sinusoidal model of Lab 3 problem 3.4.1, and model analysis in Lab 8). They were tasked with smoothing the data and discussing the new smooth model and how a solar energy collection system could be built that allowed reality to reflect what was happening numerically (that is to say, adding storage units that would accommodate local minima in solar radiation levels). They were then tasked with determining just how big a solar panel would be needed to energize a typical house for the whole year.

They then turned to an economic analysis, since the challenge is Make Solar Energy Economical. Specifically, they had to determine the break-even cost, over 15 years, for a solar panel in North Carolina given the radiation levels analyzed in the first part of the assignment and given energy consumption and costs as provided by an online resource. Finally, they had to compare this value with the actual costs per area of different solar panels and present the information graphically.

\section{Stage 6 (Analysis and Reflection)}

Finally, with all the data and graphs and resources in hand, students had to reflect on what they had learned. Specifically, the assignment stated:

The preceding exercise is just one way computational tools - including visualizing data sets and determining the coefficients of scientifically reasonable models - can be used to provide scientists and engineers with the information needed to start tackling some aspects of the Grand Challenges. For this part of the lab, your task will be to write about the Grand Challenge of Make Solar Energy Economical.

In particular, now that you have performed some quantitative work, you can once again reflect on the Challenge as a whole and on some specific elements of it given your work and conclusions in the first part of this assignment. This reflection should be at least two pages, single spaced, in LATEX, using the traditional font sizes and margins you have been using all semester. In it, you must speak to the following questions:

- How were the processes used in the programming assignment in this lab able to illuminate some aspect of the "Challenge" part of making solar energy economical? 
- How might the specific analyses performed here contribute to the solution of the Challenge or possible help re-define the Challenge?

- What are the limitations of the analysis? What other factors and disciplines should be considered to have a more complete picture?

Also, in Homework 4, you were asked to present your interpretation of the challenge. In the time since, describe how aspects of that interpretation may have been strengthened, weakened, or simply changed based upon any further research or work you have done, including this assignment.

These papers were collected, and the teaching assistants graded them for completeness and then ranked their top 3 on subjective measures.

\subsection{Fundamentals of Digital Signal Processing}

Fundamentals of Digital Signal Processing (ECE 381) is an advanced (junior/senior-level) elective for students majoring in electrical and/or biomedical engineering. Typical enrollment is around 25 students, and in the Fall semester of 2012 there were 20 students enrolled. This course provides an introduction to the theory and applications of digital signal processing, including topics such as sampling and reconstruction, discrete-time transforms including the z-transform, discrete-time Fourier transform, and discrete Fourier transform, and the analysis and design of FIR and IIR filters.

For several years, the course has had a laboratory component in which students completed both software- and hardware-based exercises. These exercises have included the simulation of a plucked string, the design of a basic speech analysis (vowel classification) system, and the implementation of a real-time electric guitar tuner. Although these projects have generally been very well-received, we continued to search for projects that would highlight even broader applications of signal processing that could illustrate how the field of signal processing could contribute to the solution of global and societal challenges.

The theme chosen for the Fall 2012 semester was Reverse-engineer the brain. This theme was selected, in part, because it was likely to appeal to our students' interests and we could leverage local expertise (e.g., the Biomedical Engineering department in which several faculty conduct research closely related to the challenge topic, the Duke Institute for Brain Science which brings together researchers from across the university with an interest in better understanding the brain).

The stated goals of the project, as described to students at the outset were four-fold:

1. To gain a multidisciplinary understanding of the societal importance of this challenge.

2. To be able to identify ways in which signal processing can interact with other areas of expertise (e.g., neuroscience) to advance our understanding of the brain.

3. To apply signal processing tools and techniques to investigate a fundamental question about brain functioning. 
4. To gain a greater understanding of how signal processing can inform solutions to the challenge, including its limitations, possible fruitful collaborations with other disciplines, and future challenges (including non-technical ones).

The EGC framework was implemented as follows:

\section{Stage 1 (Overview)}

The project began (in the $12^{\text {th }}$ week of the semester) with a broad, interdisciplinary introduction to the NAE Grand Challenge: Reverse-engineer the brain. Clearly, this challenge encompasses many questions, techniques, and disciplines. So, the project focused initially on exploring issues related to brain-computer interfaces (BCI).

In the first project assignment, students were assigned several readings to familiarize them with the NAE Grand Challenges, generally ${ }^{1}$, and the Reverse-engineer the brain challenge, specifically ${ }^{7}$. Other materials (short videos and an article ${ }^{8,9}$ ) provided a basic background of BCI. Finally, students were directed to the website of Dr. Greg Appelbaum, a neuroscientist and faculty member from the Duke Institute for Brain Sciences, who conducts research on brainbehavior relationships and in particular how the brain supports processes of vision and attention.

The assignment was to read the provided materials and submit three or more questions for Dr. Appelbaum, who was the guest speaker in class the following week. Dr. Appelbaum's lecture, "Reverse Engineering the Brain: Primer for EEG and BCI approaches", provided an introduction to electroencephalography (EEG) signal and data collection, and brain computer interface techniques. In addition to providing some general technical background and a discussion of some of the challenges often encountered, Dr. Appelbaum discussed some of his research on visual attention. The lecture was followed by a lively Q\&A session.

\section{Stage 2 (Restatement) and Stage 3 (Relation to Engineering)}

Following the activities of Stage 1, students were asked to describe the Grand Challenge in their own words and to think more specifically about the role that signal processing could play in investigating this Grand Challenge. Two more articles on BCI were assigned. ${ }^{10,11}$ Unlike the readings from Stage 1 which presented BCI from a general, non-engineering perspective, these articles (one from the IEEE Signal Processing Magazine, the other which described a MATLAB/SIMULINK model of BCI) were more discipline-focused.

The assignment was two-fold. First, students were asked to restate the Grand Challenge of Reverse-engineer the brain in their own words to the Board of Directors of a hypothetical company interested in developing signal processing algorithms for BCI applications. Second, the students were told to write a brief memo to the Board describing a possible application of BCI and to discuss the potential for success (and failure) from a signal processing perspective. Specifically, the memo had to include: 1) identification of an application (either hypothetical, or one actually being proposed or currently in production), 2) a critical evaluation of the application, including its technical soundness, 3) a discussion of related ethical and/or safety issues, and 4) their final recommendation/summary of the product. 


\section{Stage 4 (Content, Tools, and Techniques)}

Stage 4 activities actually took place throughout the semester, as the tools and techniques needed to understand the signal processing taking place in $\mathrm{BCI}$ applications were presented in lectures and reinforced in homework and earlier laboratory assignments. In anticipation of the themebased project near the end of the semester, references were made throughout the semester, as appropriate, to link a concept (such as the design and behavior of a notch filter) to its possible application (such as removal of $60-\mathrm{Hz}$ noise from an EEG signal).

\section{Stage 5 (Application)}

In Stage 5, students were asked to analyze a set of real EEG data collected at the Wadsworth Center at the New York State Department of Health by Dr. Jonathan Wolpaw and made publically available via the BCI Competition 2003. ${ }^{12}$ Specifically, students were given EEG data collected from several subjects who were asked to stare at a computer monitor and then move a cursor to a displayed target that was presented in one of four locations (of varying height), randomly determined for each trial. For the task, the BCI algorithm was designed to use two primary features for moving the cursor: mu waves (i.e., oscillations at $\sim 8-12 \mathrm{~Hz}$ ) and beta waves (i.e., oscillations between $18-24 \mathrm{~Hz}$ ). While the amplitude of these waves is not subject to direct conscious control, the participants could learn to modulate them unconsciously to move the cursor to a target.

The students were provided with a set of training data (for which the actual target location was known) and a set of test data (for which the targets were unknown to the students). The assignment was to develop an algorithm for predicting the target location to which the subject was trying to move the cursor. Successful algorithm development required students to determine parameters (e.g., energy in a narrow frequency band) that were useful in discriminating between target locations. Students had to apply concepts such as the calculation of the FFT and filter design. Once a set of parameters were identified, students had to devise an algorithm using those parameters to predict target location. They were able to test and refine their algorithm on the training data. Once they were satisfied with the algorithm's performance, they generated a set of predictions for the test (blind) data and submitted it to the instructor for scoring. The instructor then compared the predictions to the true target locations (known only to the instructor) and calculated the accuracy of each student's algorithm. The top performers were recognized in class and given the opportunity to describe their algorithm.

\section{Stage 6 (Analysis and Reflection)}

For the final stage, students reflected on what they had learned through the various activities of the project. This took place as a class discussion in which students were invited to talk about the applications of BCI they had investigated for the memo of Stage 3, the challenges they experience and insight they had after performing the data analysis of Stage 5, and unanswered questions that they had. The students were very engaged in the discussion and, anecdotally, several students expressed interest in pursuing the project in greater depth, either through additional data analysis or getting in touch with Dr. Appelbaum to identify possible research opportunities. 


\section{Impact on Student Motivation and Learning}

A key finding within the current engineering education literature is that exposure to real-world applications - especially when presented in an active, experiential learning environment increases both student interest and pedagogical effectiveness. ${ }^{13-16}$ This idea of contextual learning, defined by Karweit as learning designed so that students can carry out activities and solve problems in ways that reflect the real-world nature of such tasks, ${ }^{17}$ is based on cognitive learning theory and current research in cognitive psychology and neuroscience. Such research suggests that initial learning, transfer of that learning to other contexts, and retention of learned material is facilitated when concepts are presented in a familiar or relatable context. ${ }^{17-20}$ That is, students are motivated when learning has meaning.

We hypothesize that the use of the EGC framework will have salutary effects on student motivation and learning and have implemented the following assessment strategy:

Student Motivation. To examine how participation in engineering courses grounded in the Grand Challenges support motivation and subsequent academic achievement, we are assessing students' perceived competence in and interest/value for engineering. Perceived competence is being measured using the 5-item self-efficacy scale from the Patterns of Adaptive Learning Survey (PALS). ${ }^{21}$ A sample item includes 'I'm certain I can master the skills taught in my engineering courses.' Interest/value is being assessed in terms of students' enjoyment and value for engineering using an 8-item interest/value scale developed Linnenbrink-Garcia and colleagues. ${ }^{22}$ Sample items include 'Engineering is exciting to me' (enjoyment) and 'Engineering is practical for me to know' (value). Pilot data obtained from Duke undergraduates indicated that items from both scales are highly reliable (perceived competence: $\alpha=0.91$; interest/value: $\alpha=$ 0.93). Gender differences will be of particular interest, as we hypothesize this approach will be particularly appealing to women.

Student Learning. A primary hypothesis of the project is that student understanding of course material will improve as a result of increasing the real-world relevance of course concepts. Learning objectives and measurable outcomes were defined for all engineering course at Duke University in the Fall of 2007. Student performance will be quantified by calculating individual achievement scores. These calculations will be based on student performance (grades) on specific exam questions, homework, laboratory reports, and other course assignments. For each of those assignments, students are rated as non-proficient (0), proficient (1), or highly proficient (2). Historic data is available for all courses (Fall 2007 - present) which provides a comprehensive baseline to which student performance in the EGC framework version of the course can be quantitatively and directly compared.

Program Outcomes. Another hypothesis is that use of the EGC framework will increase students' awareness of the global and societal implications of engineering, understanding of contemporary issues, and the importance of life-long learning (ABET criteria h, i, and j). Gains in these areas will be quantified by having students rate the degree to which the course increased their ability to achieve the ABET a-k objectives on an end-of-semester survey. In a previous project, a multiple regression analysis of similar data was successfully used to show statistically significant improvements in these measures when investigating the redesign of the introductory ECE course. ${ }^{23}$ Historic data exists for more than five years and will be used as a baseline to which to compare EGC student responses. 
In the 2011-12 academic year, baseline data were collected from 387 students enrolled in engineering courses (including the two "intervention" courses in which the EGC framework was implemented in Fall 2012). Students were asked to complete at 15-minute survey at the beginning and end of the semester. In addition, course grades were collected. A series of hierarchical multiple regressions were conducted to examine various motivational variables (perceived competence, personal interest, situational interest, and epistemic beliefs) as predictors of learning outcomes, after controlling for other factors suspected to influence learning outcomes (e.g., prior ability and gender). The results were consistent with other published studies:

Perceived competence was positively associated with grades and achievement of course learning objectives; personal interest was positively associated with course learning objectives and ABET outcomes; situational interest (catch, hold-feeling, and hold-value) was positively related to grades, ABET outcomes, and learning objectives. For epistemic beliefs, simple/certain knowledge was negatively related to grades and course learning objectives, justification by authority was positively related to ABET outcomes and course learning objectives, and personal justification was positively related to ABET outcomes. Taken together, these findings highlight the importance of supporting motivational and epistemic beliefs to support learning and engagement in engineering classes.

Further analysis of the baseline data examined how students’ perceptions of the classroom supported their situational interest, after controlling for their initial interest. Three aspects of the classroom were assessed: autonomy support (professor provides opportunity for choice), connection to real life (e.g., solving real-world problems); and active involvement. Not surprisingly, all three variables significantly predicted situational interest. These results were taken into consideration as specific activities were developed for the implementation of the EGC framework in each of the pilot courses in Fall 2012

Data, as described above, were collected in the Fall 2012 semester. At this time, the analysis of these data is ongoing.

\section{Conclusions}

The EGC framework was successfully implemented in two different engineering courses using two different Grand Challenge themes. Baseline data suggest that this approach has the potential for making a significant impact on student learning and motivation, and anecdotal evidence from the pilot implementation in Fall 2012 suggests that the EGC framework approach had a positive effect. More rigorous analysis of assessment data will be conducted in order to quantify this effect.

Future plans include the implementation of the framework in two additional courses in the Spring 2013 semester (in Civil and Environmental Engineering). The framework will also be implemented in EGR 103L and ECE 381 again in the Fall 2013 semester, with modifications based on the pilot offering.

\section{Acknowledgements}

This work was supported by NSF grant \#DUE-1141073. 


\section{Bibliography}

1. "Introduction to the Grand Challenges for Engineering”, engineeringchallenges.org, 7 January 2013, <http://www.engineeringchallenges.org/cms/8996/9221.aspx>

2. Educating the Engineer of 2020, Washington DC: National Academies Press, 2005, National Academy of Engineering.

3. Coyle, E.J., Jamieson, L.H., and Oakes, W.C. (2005). EPICS: Engineering Projects in Community Service. International J. of Engineering Education, 21(1), 139-150.

4. “Homework 4: Grand Challenge: Make Solar Energy Economical”, pratt.duke.edu, 7 January 2013, $<$ http://classes.pratt.duke.edu/EGR103F12/LabManual/HW04_F12.pdf>

5. “Lab 4: Loops and Plots”, pratt.duke.edu, 7 January 2013, $<$ http://classes.pratt.duke.edu/EGR103F12/LabManual/LAB04_F12.pdf>

6. “Lab 10: Analyzing Solar Energy Data”, pratt.duke.edu, 7 January 2013, $<$ http://classes.pratt.duke.edu/EGR103F12/LabManual/LAB10_F12.pdf>

7. “Reverse-engineer the brain”, engineeringchallenges.org, 7 January 2013, $<\underline{\text { http://www.engineeringchallenges.org/cms/8996/9109.aspx }>}$

8. "Brain machine interfaces” and "Crossing Disciplines”, nature.com, 7 January 2013, $<$ http://www.nature.com/nature/focus/brain/video/index.html>

9. Fazel-Rezai, Reza (2009). "P300-Based Speller Brain-Computer Interface” in Recent Advances in Biomedical Engineering, Ganesh R Naik (Ed.), InTech, Available from: http://www.intechopen.com/books/recentadvances-in-biomedical-engineering/p300-based-speller-braincomputer-interface

10. Rao, P.N. R., and Scherer, R. (2010). Brain Computer Interfacing. IEEE Signal Processing Magazine, July 2010, 148-152.

11. Guger, C. et al. (2009). How many people are able to control a P300-based brain-computer interface (BCI)? Neurosci. Letters, 462, 94-98.

12. “BCI Competition II, Data set IIa: Self-regulation of mu- and/or central beta-rhythm”, 7 January 2013, $<$ http://www.bbci.de/competition/ii/>

13. Shuman, L.J., et al. (2002). “The future of engineering education," in Proc. $32^{\text {nd }}$ Annu. Frontiers in Education Conf., Boston, MA, Nov. 2002, vol. 1, pp. T4A-1-T4A-15.

14. Olds, B., and Miller, R. (2004). The effect of a first-year integrated engineering curriculum on graduation rates and student satisfaction: A longitudinal study. J. Eng. Educ., 93(1), 23-36.

15. McLellan, J. H., et al. (1996). Using multimedia to teach the theory of digital multimedia signals. IEEE Trans. Educ., 38(3), 336-341.

16. Munson, C.C. (1995). "Elements of a new Electrical Engineering curriculum at Illinois: A shift from circuits to signal processing,” in Proc. IEEE Int. Symp. Circuits and Systems, Seattle, WA, vol. 1, pp. 1Sf-4Sf.

17. Karweit, N. (1998). “Contextual learning: A review and synthesis”, in A.M. Milne (ed.), Educational reform and vocational education (pp. 53-84). Washington, DC: U.S. Department of Education, Office of Educational Research and Improvement, National Institute on Postsecondary Education, Libraries, and Lifelong Learning.

18. Caine, R.N., and Caine, G. (1997). Education on the edge of possibility. Alexandria, VA: Association for Supervision and Curriculum Development. 
19. Lave, J. and Wenger, E. (1991). Situated learning: Legitimate peripheral participation. Cambridge, UK: Cambridge University Press.

20. Bransford, J.D., Brown, A.L., and Cocking, R.R. (1999). How People Learn: Brain, Mind, Experience and School. Washington, DC: National Academy Press.

21. Midgley, C., Maehr, M. L., Hruda, L. Z., Anderman, E., Anderman, L., Freeman, K. E., et al. (2000). Manual for the Patterns of Adaptive Learning Scales (PALS). Ann Arbor, MI: University of Michigan

22. Linnenbrink-Garcia, L., Durik, A. M., Conley, A. M., Barron, K. E., Tauer, J. M., Karabenick, S. A., and Harackiewicz, J. M. (2010). Measuring situational interest in academic domains. Educational and Psychological Measurement, 70, 647-671.

23. Huettel, L.G., Brown, A.S., Coonley, K.D., Gustafson, M.R., Kim, J., Ybarra, G.A., and Collins, L.M. (2007). Fundamentals of ECE: A Rigorous, Integrated Introduction to Electrical and Computer Engineering. IEEE Trans. Education, 50(3), 174-181. 